\title{
High Boron Silicon Nanotubes Combined with Tai Chi Exercise Rehabilitation Therapy in the Treatment of Knee Arthritis Patients
}

\author{
Guang Ma (iD) \\ Department of Physical Education, Lvliang College, Lvliang, Shanxi 033001, China \\ Correspondence should be addressed to Guang Ma; 37017@llhc.edu.cn
}

Received 6 August 2020; Revised 17 September 2020; Accepted 12 October 2020; Published 31 October 2020

Academic Editor: Tifeng Jiao

Copyright (C) 2020 Guang Ma. This is an open access article distributed under the Creative Commons Attribution License, which permits unrestricted use, distribution, and reproduction in any medium, provided the original work is properly cited.

\begin{abstract}
Tai Chi exercise is gentle, convenient, and easy to learn. It is more economical than traditional medical treatments, and it is regarded as the first choice for rehabilitation therapy by patients with knee arthritis. This article aims to study Tai Chi exercise rehabilitation therapy combined with high boron silicon nanotubes to treat knee arthritis patients. This article mainly introduces the treatment of knee arthritis patients with Tai Chi, which is reflected in the improvement of patients' walking ability and stability, and explores a three-dimensional motion model to provide better help for patients with knee joints. The article uses data mining methods to collect data on the gene expression of human knee joints and analyzes the causes of knee arthritis caused by its internal structure. The experimental results of this paper show that, under Taijiquan exercise rehabilitation treatment, the time needed by knee arthritis patients to get up and run is reduced by $14 \%$, the standing time of one leg is significantly improved, the fall rate is reduced by $13 \%$, and the body's static balance ability is improved.
\end{abstract}

\section{Introduction}

1.1. Background and Significance. Knee osteoarthritis is a chronic deformable arthritis that is most common for the elderly. When the knee arthritis is severe, the bones will disappear. As a result, the friction between the bones and bones increases, which makes the patient's walking, daily activities, and other obstacles worse. Tai Chi exercise is an effective treatment for knee arthritis proposed by the American Rheumatism Association. Tai Chi emphasizes flexibility and requires practitioners to keep in touch and repeat it all the time but not monotonously. According to a large number of studies, Tai Chi exercise can strengthen the body, mainly reducing the pain on the sick body, improving the flexibility of the body's joints, and helping the treatment of patients with knee arthritis; it can also purify the mind.

The importance of Tai Chi for practitioners has gradually evolved from the initial five strong bodies to longevity and has the health and other heart and lung functions of Tai Chi. The most special aspects of Tai Chi are the control of the balance mechanism and the treatment of knee arthritis. It has received more and more attention and learning from the elderly.
1.2. Related Work. The Tai Chi exercise proposed by Miller and Taylor-Piliae is beneficial to cognitive and physical functions and may affect the safe driving performance of the elderly. The main purpose of this research is to compare the cognitive processes and physical functions of elderly Tai Chi practitioners related to safe driving with standard reference values; the second purpose is to study the relationship between Tai Chi exercise habits, cognitive processes, and physical functions related to safe driving performance and explore potential predictive indicators of safe driving performance. However, this research is solely related to safe driving, which makes the research not very comprehensive [1]. Lee et al. studied the effect of extracorporeal shock wave therapy on pain and function in patients with degenerative knee arthritis. 20 patients with degenerative knee arthritis were divided into conservative physical therapy group and extracorporeal shock wave therapy group. Both groups received conventional conservative physical therapy. Then, extracorporeal shock wave therapy plus extracorporeal shock wave therapy was received. However, because there were not enough control groups during the study, the research results were not very accurate [2]. Janssen et al. proposed that rehabilitation after treatment of non-small cell 
lung cancer (NSCLC) has a beneficial effect on exercise capacity, but there is limited insight into the impact on quality of life (QoL) and fatigue, and its purpose is to examine the treatment of NSCLC stages I to IIIa of adult patients and pulmonary rehabilitation (PR) results related to fatigue, QoL, and exercise capacity. However, due to the excessive number of cells, the conclusion is not very accurate [3].

1.3. Main Content. This paper combines the finite element model, the dynamics of the knee joint, and the movement data of Tai Chi and reveals the regularity of the change of the tension of the knee joint of Tai Chi. In order to understand the impact of knee-knocking movement on the internal structure of the knee joint, it will provide theoretical support for the practice of Tai Chi and physical rehabilitation training in the future.

According to the establishment of the Comprehensive Research Institute, this article randomly divides elderly patients with knee deformity arthrosis as a control group into three-month Tai Chi practice groups. The functional ability of the subjects who walked to the knee joint before and after intervention was tested. A motion capture system is designed to obtain the parameters of the rebound force of the test subject's feet and the ground, then calculate the stability force according to the formula, and finally compare the data with the actual data.

\section{Treatment of Knee Arthritis}

2.1. Principal Component Analysis. In this document, a fivepart scale is used to rank the impact indicator $n(n=8)$. When the score is " 5 points", this means that the indicator is needed; the score of " 4 points" means that it is more needed; and the scores of " 3 points" and " 2 points" mean that it is not needed. When the score is " 1 point", this means that the indicator is not needed at all. It is planned to use the principal component analysis method to calculate the weights and obtain the specific weight ratio [4].

The main component analysis, abbreviated as PCA, is an important method of multifactor statistical analysis. It collects the information propagated by a set of variables in some comprehensive indicators (principal component, PC) and reduces the dimension of the dataset, which simplifies the problem. The usual mathematical process is to linearly combine the initial index $n$ into a new comprehensive index. The most commonly used method is to express the change of $f_{1}$ (the first linear combination selected, that is, the first comprehensive indicator), that is, variable $1, F_{1}$, contains more information. Therefore, $F_{1}$ is the variable with the greatest variability among all linear combinations, because $F_{1}$ is called the first principal component. However, the information of the initial $P$ index is not enough to be represented by the first principal component $F_{1}$; then the second linear combination $F_{2}$ can be selected to represent it. In order to effectively reflect the initial information, the existing information of $f_{1}$ does not need to appear in $f_{2}$, expressed in mathematical language as $\operatorname{Cov}\left(f_{1}, f_{2}\right)=0$; then, $f_{2}$ is called the second main component; the third, fourth, and first major components can be manufactured to scale. The first and the main components can be manufactured in proportion [5].

The mathematical model of principal component analysis can be derived from the following three formulas:

$$
\begin{aligned}
& F_{1}=a_{11} X_{1}+a_{21} X_{2}+\cdots+a_{n 1} X_{n}, \\
& F_{2}=a_{12} X_{1}+a_{22} X_{2}+\cdots+a_{n 2} X_{n}, \\
& F_{k}=a_{1 m} X_{1}+a_{2 m} X_{2}+\cdots+a_{p m} X_{n},
\end{aligned}
$$

among which, $t_{i j}$ is the coefficient of each component, expressed as

$$
t_{i j}=\frac{f_{i j}}{\sqrt{\lambda_{j}}}, \quad j=1,2, L, m .
$$

$a_{i}$ is the comprehensive importance in the principal component, expressed as

$$
a_{i}=\sum_{j=1}^{m}\left(\frac{\lambda_{j}}{k}\right) t_{i j}, \quad k=\lambda_{1}+\lambda_{2}+l+\lambda_{m} .
$$

Through normalization, the final index weight $w_{i}$ is determined as

$$
w_{i}=\frac{a_{i}}{\sum_{i=1}^{n} a_{i}}
$$

Finally, the comprehensive score model of principal component analysis is

$$
Y=w_{1} X_{1}+w_{2} X_{2}+\cdots+w_{n} X_{n}
$$

Finally, an index weight value was calculated for each dimension. From the index weights, the weight ratio of Tai Chi exercise to the treatment of knee arthritis is the largest [6].

2.2. Data Mining Method. In this article, we use data mining methods. First, we collected gene expression profile data related to human knee osteoarthritis, and then we selected gene expression profiles that meet the requirements [7]. Then, we used GEO2R to obtain different expressed genes. Next, the use of DAVID for the enrichment analysis of the expressed genes in GO-comment and KEGG pathways and the use of cell landscape for the integration of different integrated genes to participate in the interaction of different biological pathways are of great research significance for network visualization, analysis, and discussion.

We selected the project type as a series and restricted the species to humans to obtain the relevant qualified series. In order to expand the search scope, in addition to searching for keywords in the gene expression database, an additional search was also conducted in the PubMed library, containing additional filter conditions: (1) search contains grid term, osteoarthritis; (2) document language should be English [8].

Since the experimental object and experimental platform are selected from different datasets, the cell type, control group type, and data type selected by the experiment should 
be further checked. The specific classification condition is defined as follows: the source of the abandoned sample is not joints or fibers, such as stem cells, excluding the normal control group without OA or with no record. Screening according to experimental methods, in addition to "RT-PCR expression profile", we retained only high-performance analytical techniques (sequencing or "fine adjustment"). At the same time, we selected the data type as the gene expression profile, in addition to noncoding RNA, methylation, and other expression profiles: "noncoding pattern of RNA sequence", "methylation pattern by array (genotype plate)". Finally, the concept of meaningless records was abandoned, and the information of the data set was confirmed. The meaningless record concept confirms the information in the dataset [9].

2.3. Quantitative Research Method of Knee Motion. In order to evaluate the stability of the test object, it is necessary to calculate the value of the horizontal force required to break the balance of the test object. This evaluation method is based on the stable margin defined by Koozekanani et al. [10]. The stability margin refers to the distance where the COP is the smallest from the support surface. If the remaining amount is zero, then the human body will be in balance, and the remaining stable amount is proportional to its own physical ability. To make the stability margin zero, you only need to add a to the system, and then the formula is

$$
C_{\mathrm{d}}=\overrightarrow{d_{\mathrm{cp}}} \times \overrightarrow{F_{\mathrm{r}}}
$$

$\overrightarrow{d_{\mathrm{cp}}}$ refers to the smallest distance to COP from the supporting surface, and $\overrightarrow{F_{\mathrm{r}}}$ refers to the magnitude of the rebound force formed with the ground. In addition, if there is a virtual enough foot to compensate for the horizontal force the system bears, then the torque created by a single virtual horizontal force may destroy the stability of the human body. To make the virtual horizontal force sufficiently sensitive in the center, the lever arm $\left(h_{\mathrm{CM}}\right)$ is selected as the distance of the center of gravity from the support surface. Therefore, the cause of unstable arthropods can be expressed as the following formula:

$$
C_{\mathrm{d}}=\overrightarrow{h_{\mathrm{CM}}} \times \overrightarrow{F_{\mathrm{D}}} \text {. }
$$

We further derived

$$
\overrightarrow{F_{\mathrm{D}}}=-\frac{\left|\overrightarrow{F_{\mathrm{R}}}\right|}{\left|h_{\mathrm{CM}}\right|} \cdot \overrightarrow{d_{\mathrm{cp}}}
$$

The area surrounded by the ground projection positions of the three marked points of the foot (the first middle finger, the fifth middle finger, and the heel point) is defined as the supporting surface. Calculate the magnitude of the virtual horizontal force on both sides of the support surface, and use the minimum value to describe the stability characteristics of the test object. The minimum virtual force is the value of the force required for human body instability and is directly related to human body stability [11].
The direction of $\overrightarrow{F_{\mathrm{D}}}$ indicates the direction in which the subject is most likely to lose balance and is related to the vector indicating the average direction characteristic of the foot. The vector $\overrightarrow{O_{f}}$ of the average foot orientation coincides with the direction of the left foot and defines a positive direction that destabilizes the human body. The angle of this vector is counterclockwise. However, in this study, the amount of unstable force is mainly used to measure the body's ability, regardless of the direction of the force, to maintain a balance with the falling force [12]. The smaller the value of the instability is, the easier the personal stability is destroyed.

This method evaluates dynamic balance, and dynamic performance balancing elements vary according to the shape and size of the bearing surf, so the difficulty of balancing can be fully understood. At this point, using this model to properly evaluate the stability of patients with deformed knee arthritis will help to optimize the evaluation and optimize the effect of Taijiquan education on the entire knee arthritis intervention program [13].

\section{Experiment of Tai Chi on Knee Joint}

3.1. Parameter Collection. Before starting the experiment, calibrate the Weikang and Kistler 3D force platform and 3D calibration system in the experimental area. All people wear experimental clothing (ordinary cotton shorts, vests, cotton socks, and rubber shoes) prepared in the laboratory and use anthropometry to measure the basic characteristics of the human body such as weight and height. The requirements and basic ideas of the experiment are roughly clear. The subject will use 47 infrared reflections attached to the corresponding positions on the body surface to determine the head and torso. Before the formal exercise test, the upper and lower limbs are doubled and statistical data are collected as required [14]. In this study, Weikang cameras were used for sampling. The frequency is $80 \mathrm{~Hz}$, and the sampling frequency of the Kistler 3D power platform is $980 \mathrm{~Hz}$. Typical movement requires an individual to walk 12 meters at a comfortable body speed. The corridor contains two 3D power boards at the same height as the ground. Of course, All experimental subjects used in this article adopt a natural forward approach. When two force plates measure one foot of data and the two data are continuously in two data levels, the data will be judged as valid. This requires the experimenter to be familiar with and master the infrared motion system and the movement. The process can ensure that three of the collected data items are valid. Through the fourthorder low-pass filtering, the ground bounce signal of the dynamic signal can be collected, and the intensity of the frequency cutoff is $24 \mathrm{~Hz}$ and $54 \mathrm{~Hz}$, respectively [15].

3.2. Knee Movement Experiment. The object of the experiment is a Tai Chi player with nearly 6 years of experience. After a combination of medical diagnosis and acquisition of knee MRI images, there were no knee injuries in the past 3 years. The measurement movement is the representative movement of Tai Chi, the steps around the knees and arms. 
The type of exercise is similar to walking and jogging. This is also forward and periodic movement, which is convenient for comparative analysis [16].

The experimental device used a VICON motion analysis system (VICON MX, Oxford Measure, UK) and 8 VICON MX-40 cameras, and the acquisition frequency was set to $100 \mathrm{~Hz}$. In addition, two 3D Fort boards (9287B, Kistler Corporation, Switzerland) were used to record ground reaction force data at a frequency of $1000 \mathrm{~Hz}$. The subject's right knee joint was scanned using MRI (1.5T, Siemens, Germany). The collection process is shown in Figure 1.

Place a nonslip blanket on the surface of the force measurement platform to prevent personnel from knowing the specific location of the force platform and avoid the visual impact of the power platform to properly adapt to the theme rhythm [17]. Adjust the reflective ball to the bottom of the person, with a diameter of $14 \mathrm{~mm}$. The location of the coupling point is based on the default back-end model visual 3D: the pivot point includes the posterior iliac point and the anterior superior iliac spine point on both sides; the rabbit's waist has large rotor points on both sides the hub of the thigh ring includes big rotor points on both sides and inner and outer points of knee joints on both sides; one heating plate is connected to both sides of the thigh, and four points are connected to each heating plate. The points for monitoring the thigh-calf ring includes the middle of the knee joints on both sides and the lateral point, the medial and lateral points of the ankle joint on both sides of the ankle joint, and a thermoplastic steel plate on each side of the lower leg. Each thermoplastic board is connected to four points as the monitoring points for the calf connection; foot connection points are the first and fifth points of the column, toe point, and heel point [18].

After the statistical data collection is complete, start collecting other mobile data. At the beginning of the order, the subject drove at the speed he felt most comfortable, began to move within 5 meters from the power platform, and stopped at a distance of 2 meters after reaching the power platform (no human intervention around). The bottom right edge of the center of the power platform area is used as single valid data (because the data is collected in the center of the platform for each movement, walking, jogging, knee joint). Three valid data items are collected. Use specific software to test each energy and process four to five times, and then average the data [19]. The kinetic data is processed by $20 \mathrm{~Hz}$ low-pass filtering. Once the right foot enters the force platform until the side legs leave the force platform, the statistical period is expressed as a percentage.

\section{Knee Arthritis Treatment Analysis}

4.1. Nanotube Preparation Analysis. The ABA11 molecule was dissolved in ethanol, and the prepared solution was allowed to stand at room temperature for $3 \mathrm{~d}$. At this time, the ABA11 molecule self-assembled to form nanotubes, and these nanotubes had uniform diameters. In the enlarged image of the transmission electron microscope (TEM), the diameter and wall thickness of the nanotubes can be clearly seen. The outer diameter of the nanotube is

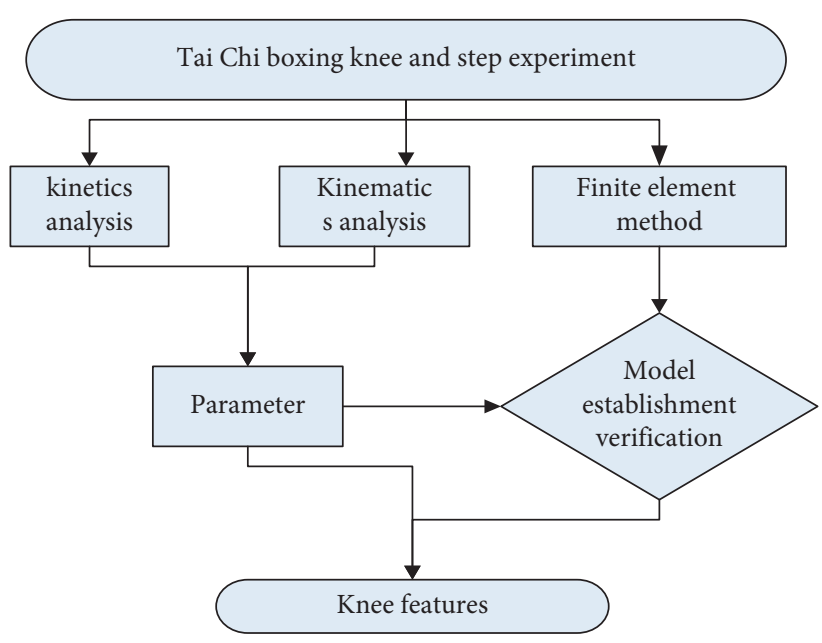

Figure 1: Flowchart of knee movement data collection.

about $32 \mathrm{~nm}$, and the thickness of the nanotube wall is about $8 \mathrm{~nm}$. The length of a single elongated ABA11 molecule estimated by ChemDraw is about $2.7 \mathrm{~nm}$; that is, the thickness of the tube wall is about twice the length of the ABA11 molecule. However, cross-sectional analysis of atomic force microscope (AFM) images shows that the outer diameter of the nanotubes is about $656 \mathrm{~nm}$, which is inconsistent with the results of TEM testing [20]. At this time, we need to pay attention to the working mechanism of AFM. AFM is realized by the interaction force between the needle tip and the sample during the scanning process. When the size of the needle tip is not negligible compared to the sample size, the amplification effect of the needle tip must be considered. In other words, the width of the needle tip was incorrectly included in the sample size during the scan. We captured an AFM image of a single nanotube, and a bundle of nanotubes were arranged parallel to each other, so that the true diameter value can be calculated. Assuming that each side of the nanotube has a needle tip amplification effect and its amplification value is $a$, the needle tip amplification effect only acts on both sides of the sample, and it is assumed that the actual outer diameter of the nanotube is $d$ [21]. According to the crosssectional analysis diagram of AFM, the outer diameter of a nanotube should include the diameter of the actual nanotube and the part enlarged by the needle tip on both sides; then, we can get the following equation: $2 \mathrm{a}+d=60 \mathrm{~nm}$, four. The width of adjacent nanotubes can be expressed as $2 a+4 d=146 \mathrm{~nm}$. Therefore, the outer diameter value of the nanotube can be derived, that is, $d=26.6 \mathrm{~nm}$. The calculated value and the value obtained from the TEM image can be well matched. In addition, the height of the nanotubes obtained by AFM cross-sectional analysis was also $286 \mathrm{~nm}$, which proved that the assembly of ABA11 in ethanol was circular after drying. Moreover, during the AFM test, we just scanned the images before and after the nanotube was damaged by the needle tip, which further confirmed that the morphology we obtained was a hollow tubular structure rather than a solid rod-like structure [22]. 
4.2. Knee Movement Angle Analysis. The knee joint is at a positive angle, that is, a certain angle of overextension when walking and cushioning on the ground; the maximum angle is $16^{\circ}$; the knee joint is always in the flexed state during the subsequent support start and propulsion process; and the toe leaves at the end of the propulsion period. The maximum flexion angle of the force platform at all times is $-12^{\circ}$; the knee joint has the maximum flexion angle at $26 \%$ of the time due to the buffering effect during jogging, and the size is $-10^{\circ}$. In the knee-clap and step movements, the knee joints are mostly at a relatively large flexion angle. Most of the joints are at a large flexion angle. The curve has two peaks; the first peak is the right foot swinging phase, and the second peak is after the left lower limb touches the ground. The right lower limb stretches as shown in Figure 2.

Compared with walking and jogging, the range of motion of the knee joint in knee-knocking movements is larger [23]. The range of motion of the right knee at different angles is shown in Figure 3. In the previous studies on jogging and walking, there has been a clear division of the phase of the movement process. In this study, in order to facilitate the comparison with the knee and step movements, the three movements have been rephased.

According to the data analysis, it can be concluded that although it is the same forward movement, it is different from walking and jogging. There is a more obvious foot swinging movement in the knee and stepping movement, and the lower limb swinging is driven by the foot swinging [24]. According to the angle data of the knee joint along the vertical axis, the angle of the knee joint in the jogging and walking movements gradually transitions from the internal rotation to the external rotation. Compared with walking and jogging, knee-knocking movements have a larger range and angle of motion of the knee joint, and the knee joint is in a larger flexion position during the entire exercise. This result is consistent with previous studies [25].

\subsection{Analysis of the Influence of Tai Chi Training on Patients with Knee Arthritis}

4.3.1. Analysis of the Impact of Functional Walking Ability. As a commonly used method of evaluating functional walking ability, the timed "stand-up" test has been used in clinical and laboratory research. Through data analysis, we can get the time of sit-up test for two experimental groups (Tai Chi group, education group), as shown in Table 1:

Table 1 shows that before the start of Taijiquan intervention, the test time for the subjects in the Taijiquan group and the control group was 8.54 seconds and 8.59 seconds in the "stand-up-walk" test; at this time, there was no significant difference between the groups $(P=0.6452>0.05)$; that is, the two groups of subjects had the same level of functional walking before intervention [26].

In addition, after 3 months of Tai Chi intervention, the functional walking ability of the two groups of subjects was tested, and it was found that after 6 months of Tai Chi/ lecture intervention, the subject's timing of "stand-upwalking" changed; see Table 2 .

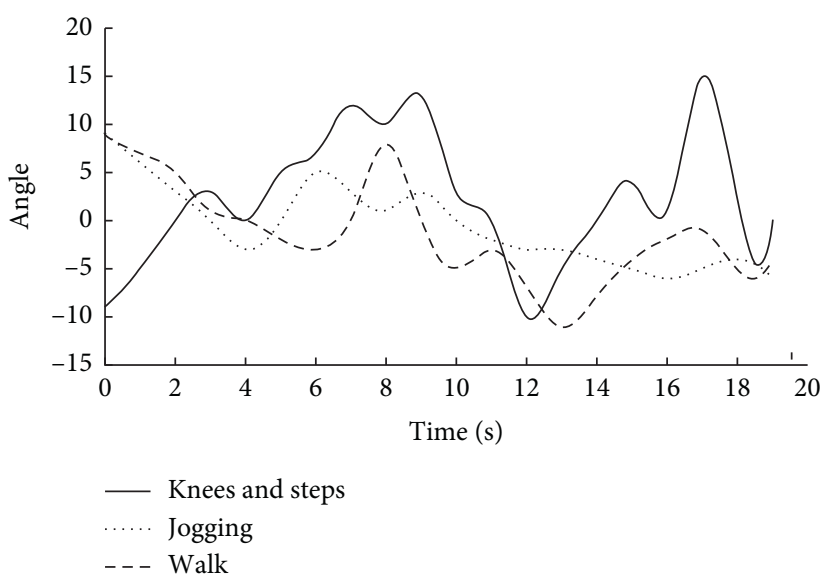

FigURE 2: Knee joint motion angle.

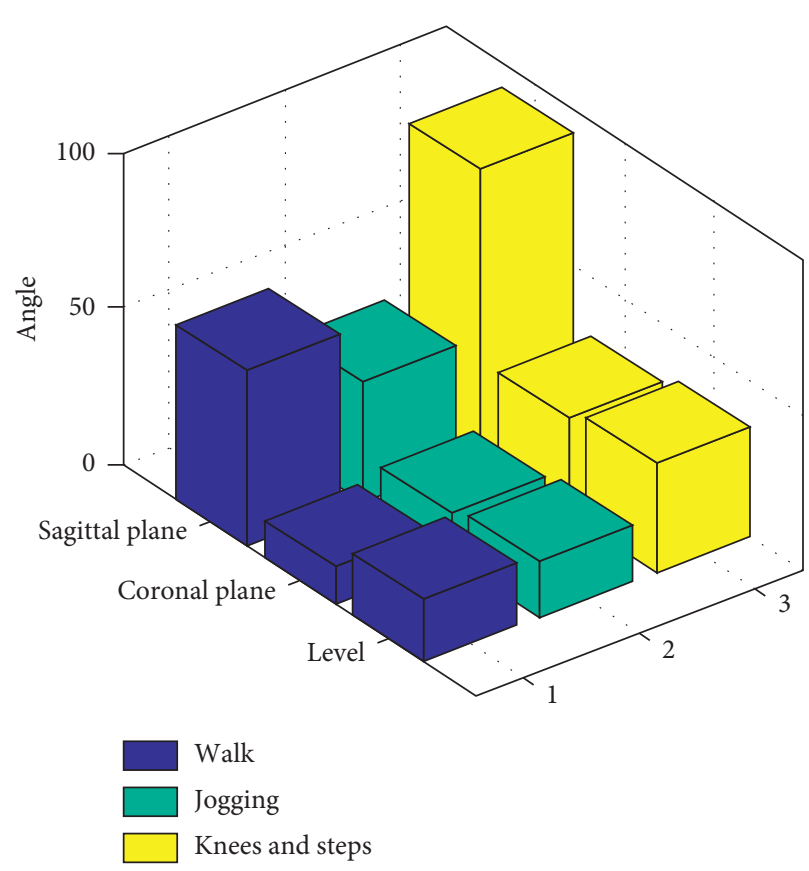

FIgURE 3: Knee motion amplitude.

TABLE 1: Comparison of timing "stand-up" before intervention.

\begin{tabular}{lccc}
\hline Project & $\begin{array}{c}\text { Tai Chi } \\
\text { group }\end{array}$ & Education group & $P$ value \\
\hline Quantity & 25 & 25 & - \\
Timing "stand-up-walk" (s) & 8.54 & 8.59 & 0.6452 \\
\hline
\end{tabular}

As can be seen from Table 2, before and after 3 months of Tai Chi intervention, the time change of the Tai Chi group to complete the timed "stand up-walk" test was extremely significant $(P=0.007<0.01)$, while the control group did not show significant changes before and after 3 months of intervention $(P=0.269>0.05)$. This shows that 3 months of Tai Chi exercises can significantly improve the functional walking ability of those with knee osteoarthritis, but 3month lectures have no improvement effect [27]. 
TABLE 2: Time change of "stand-up-walk" before and after intervention.

\begin{tabular}{lcccccc}
\hline & \multicolumn{2}{c}{ Tai Chi group } & \multicolumn{2}{c}{ Education group } & \multicolumn{2}{c}{ Tai Chi group Q } \\
Project & $\begin{array}{c}\text { Before } \\
\text { intervention }\end{array}$ & $\begin{array}{c}\text { Edter } \\
\text { intervention }\end{array}$ & $\begin{array}{c}\text { Before } \\
\text { intervention }\end{array}$ & $\begin{array}{c}\text { After } \\
\text { intervention }\end{array}$ & F/p & vs. $H$ \\
\hline $\begin{array}{l}\text { Quantity } \\
\text { Timing "stand-up- }\end{array}$ & 25 & 25 & 25 & 25 & 25 & 25 \\
walk" (s) & 8.91 & 8.73 & 8.12 & 8.33 & 1.834 & 0.941 \\
\hline
\end{tabular}

4.3.2. Analysis of the Impact of Walking Dynamic Stability Characteristics. The destabilizing force refers to the external force required to move the COP out of the boundary of the support surface. The larger the value, the greater the external force required to destroy the current stable state; that is, the more difficult the stability is to be destroyed, the more dynamic the stability is. It is good. In this study, with the help of the destabilizing force in the three-dimensional dynamic rigid body model and the six characteristic moments of the subject 's walking single support period (the right foot off the ground $T_{1}$, the left foot ground reaction force first peak $T_{2}$, the right knee flexion maximum angle $T_{3}$, the left foot ground reaction force minimum value $T_{4}$, the left foot ground reaction force second peak value $T_{5}$, and the right foot before landing again $T_{6}$ ), dynamic stability was quantitatively analyzed [28]. Before and after the intervention, the destabilizing characteristics of the two groups of subjects at the characteristic moment are shown in Figure 4.

Therefore, we further compared the destabilizing forces at each characteristic moment of the two groups before the intervention and found that the destabilizing forces at the six characteristic moments of the Taijiquan group before the intervention were $56.38 \pm 24.10,82.14 \pm 24.94,74.39 \pm 36.42$, $88.98 \pm 20.85,62.74 \pm 43.43$, and $74.57 \pm 42.33$, while the destructive forces at the six characteristic moments of the control group were $59.33 \pm 36.65,92.54 \pm 28.63$, $88.43 \pm 28.41,90.47 \pm 34.86,68.46 \pm 32.86$, and $78.36 \pm 36.73$, and there was no significant difference between the two groups. It can be seen from the data analysis that, compared with the situation before the intervention, after 3 months of Tai Chi practice, the destabilizing force values at $T_{4}$ and $T_{5}$ both increased significantly, and the increase at $T_{3}$ was extremely significant. However, in the control group, after 6 months of lecture intervention, not only there is no significant increase in destabilizing force at any characteristic moment, but an extremely significant decrease occurs at $T_{3}$. These results indicate that 3 months of Tai Chi exercises can effectively enhance the dynamic stability of KOA patients, but lecture interventions cannot [29].

4.4. Treatment of Knee Arthritis with High Borosilicate Nanomaterials. We tested the therapeutic effect of QRuPLGA-Rego-DSNPs on knee arthritis by immunohistochemistry and joint slices. The joints were histologically examined 28 days after intravenous injection. The untreated and QRuNPs groups showed significant articular synovitis and cartilage corrosion. However, the levels of synovitis and cartilage corrosion in the QRu-PLGA-Rego-

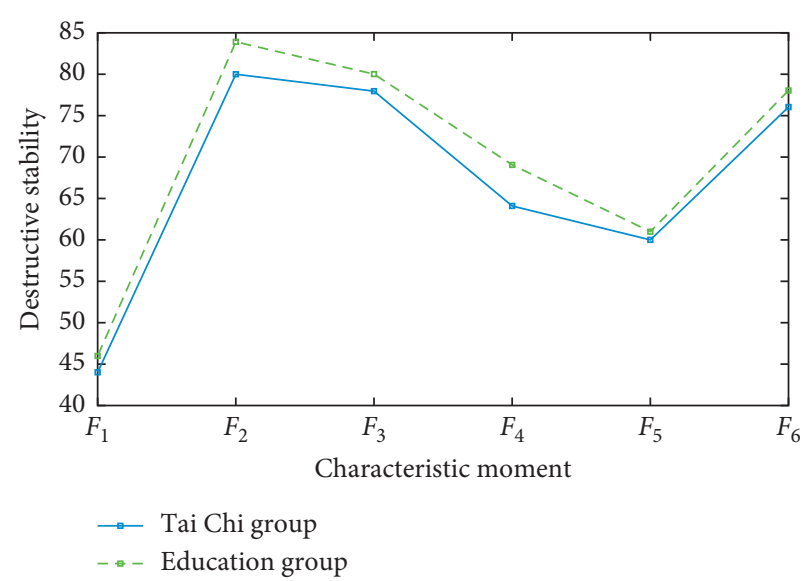

FIgURE 4: The value of the destabilizing before and after the intervention.

DSNPs and QRu-PLGA-Rego-DSNPs + laser groups were significantly improved, and the QRu-PLGA-RegoDSNPs + laser group was more effective. Inflammatory cytokines (including IL-1 $\beta$, IL-6, IL-10, and TNF- $\alpha$ ) are important factors involved in the pathogenesis of knee arthritis, and their expression levels are closely related to the inflammation activity of knee arthritis. Immunohistochemical staining showed that the expression of IL- $1 \beta$, IL- 6 , and TNF- $\alpha$ in the joints of the untreated and QRuNPs groups increased significantly (the expression levels of proinflammatory cytokines are positively correlated with the severity of inflammation). In the $\mathrm{QRu}-$ PLGA-Rego-DSNPs and QRu-PLGA-Rego-DSNPs + laser groups, the expression levels of the three proinflammatory cytokines IL- $1 \beta$, IL- 6 , and TNF- $\alpha$ were significantly reduced; QRu-PLGA-Rego-DSNPs + laser group had the best treatment effect. However, the expression of IL-10, an inflammatory cytokine that has an inflammatory effect, is opposite to the former. We observed that the expression in the untreated and QRuNPs treatment groups was significantly reduced, while in the QRu-PLGA-Rego-DSNPs and QRu-PLGA-Rego-DSNPs + laser groups, the expression was significantly increased, and in the QRu-PLGARego-DSNPs + laser group the effect is better (the expression level of IL-10 is inversely related to the degree of inflammation). These results indicate that the composite nanosystem QRu-PLGA-Rego-DSNPs + laser can effectively inhibit knee arthritis synovitis and cartilage corrosion and downregulate the proinflammatory cytokines IL- $1 \beta$, IL- 6 , and TNF- $\alpha$, while upregulating the anti-inflammatory cytokine IL-10 achieves a good therapeutic 
effect. In addition, after 28 days of treatment, from mice, their main organs for treatment for histopathology was extracted. Compared with the normal group, no obvious tissue damage was found in each treatment group, indicating that the accumulation and metabolism of composite nanoparticles in the main organs did not cause toxicity in vivo.

\section{Conclusions}

This article describes the production process of high borosilicate nanotubes and its therapeutic effect on knee arthritis. Compared with jogging and walking, the maximum pressure of the meniscus caused by knee cartilage and knee movement is smaller, and it is impossible to separate their exercise strategies. However, if the center of gravity is not correctly transferred during exercise, the greater the angle of the knee joint, the greater the load; this may cause an increase in contact tension, so there is a certain risk of knee damage.

In addition, statistical analysis of the data and model analysis show that 3 months of Tai Chi exercise can significantly improve the functional stability and antifall ability of patients with osteoarthritis. It is recommended that patients with osteoarthritis use this type of Tai Chi rehabilitation program to improve the stability of their posture. In addition, it can also be concluded that there is a moderate correlation between the stability of knee arthritis patients and walking ability, indicating that the instability index can be used to evaluate the dynamic stability of knee osteoarthritis patients.

The innovation of this article is to combine the finite element model to study the contact stress between the cartilage of the knee and the joint, the duration of the knee movement, and the lack of strength to cover the knee. A large amount of data is extracted to improve the success rate of research and avoid detours. However, due to subjective and objective constraints such as time and material conditions, the extracted results may be one-sided, with wrong analysis and success in the middle. Therefore, further analysis of recorded data and transcription data should be performed to collect more just and accurate differentially expressed genes or proteins to improve the purpose of OI pathology research.

\section{Data Availability}

The data used to support the findings of this study are included within the article.

\section{Conflicts of Interest}

The author declares that there are no conflicts of interest regarding the publication of this paper.

\section{References}

[1] S. Miller and R. E. Taylor-Piliae, "The association between Tai chi exercise and safe driving performance among older adults: an observational study," Journal of Sport and Health Science, vol. 7, no. 1, pp. 87-98, 2018.

[2] J.-H. Lee, S. Lee, S. Choi, Y.-H. Choi, and K. Lee, "The effects of extracorporeal shock wave therapy on the pain and function of patients with degenerative knee arthritis," Journal of Physical Therapy Science, vol. 29, no. 3, pp. 536-538, 2017.

[3] S. M. J. Janssen, J. J. Abbink, R. Lindeboom, and T. P. M. V. Vlieland, "Outcomes of pulmonary rehabilitation after treatment for non-small cell lung cancer stages I to IIIa," Journal of Cardiopulmonary Rehabilitation and Prevention, vol. 37, no. 1, pp. 65-71, 2017.

[4] G. Shao, Y. Lu, X. Wu et al., "Preparation and thermal shock resistance of high emissivity molybdenum disilicide- aluminoborosilicate glass hybrid coating on fiber reinforced aerogel composite," Applied Surface Science, vol. 416, pp. 805-814, 2017.

[5] G. Yang, J. Tan, H. Jin et al., "Creating effective nanoreactors on carbon nanotubes with mechanochemical treatments for high-areal-capacity sulfur cathodes and lithium anodes," Advanced Functional Materials, vol. 28, no. 32, pp. 1800595.1-1800595.9, 2018.

[6] Y. He, H. Jin, S. Qiu, and Q. Li, "A novel strategy for highperformance transparent conductive films based on doublewalled carbon nanotubes," Chemical Communications, vol. 53, no. 20, pp. 2934-2937, 2017.

[7] Y. Xiao, W. Wang, and Q. Wu, "High active and easily prepared cobalt encapsulated in carbon nanotubes for hydrogen evolution reaction," International Journal of Hydrogen Energy, vol. 45, no. 7, pp. 3948-3958, 2020.

[8] A. C. Lim, H. S. Jadhav, H. J. Kwon, and J. G. Seo, "Highloading carbon nanotubes on polymer nanofibers as standalone anode materials for Li-ion batteries," ACS Omega, vol. 4, no. 2, pp. 4129-4137, 2019.

[9] K. Ihara, H. Numata, F. Nihey, R. Yuge, and H. Endoh, "High purity semiconducting single-walled carbon nanotubes for printed electronics," ACS Applied Nano Materials, vol. 2, no. 7, pp. 4286-4292, 2019.

[10] Y. Bai, B. Shen, S. Zhang et al., "Storage of mechanical energy based on carbon nanotubes with high energy density and power density," Advanced Materials, vol. 31, no. 9, pp. 1800680.1-1800680.26, 2019.

[11] T. K. Nguyen, A. G. Bannov, M. V. Popov, J.-W. Yun, A. D. Nguyen, and Y. S. Kim, "High-temperature-treated multiwall carbon nanotubes for hydrogen evolution reaction," International Journal of Hydrogen Energy, vol. 43, no. 13, pp. 6526-6531, 2018.

[12] W. Li, J. Huang, L. Feng, L. Cao, Y. Liu, and L. Pan, "VS4 microspheres winded by (110)-oriented nanotubes with high rate capacities as sodium-ion battery anode," Materials Letters, vol. 230, pp. 105-108, 2018.

[13] C. Yu-Jen, W. Jong-Shyan, H. Chih-Chin et al., "Cerebral desaturation in heart failure: potential prognostic value and physiologic basis," PLoS One, vol. 13, no. 4, Article ID e0196299, 2018.

[14] D. M. El-Gendy, N. A. A. Ghany, and N. K. Allam, "Black titania nanotubes/spongy graphene nanocomposites for highperformance supercapacitors," RSC Advances, vol. 9, no. 22, pp. 12555-12566, 2019.

[15] A. Khuzema, A. Brammatha, and V. Arul Selvan, "Effect of home-based tai chi, yoga or conventional balance exercise on functional balance and mobility among persons with idiopathic Parkinson's disease: an experimental study," Hong Kong Physiotherapy Journal, vol. 40, no. 01, pp. 39-49, 2020. 
[16] J. Xu, S. Yu, C.-H. Goh et al., "Effect of wheelchair Tai Chi ball exercise on physical \& mental health among elderly," Medicine \& Science in Sports \& Exercise, vol. 51, no. Supplement, p. $965,2019$.

[17] H. Hamasaki, "Exercise and gut microbiota: clinical implications for the feasibility of tai chi," Journal of Integrative Medicine, vol. 15, no. 4, pp. 270-281, 2017.

[18] S. Talwar, C.-C. Chen, J. Lamberth et al., "Effects of tai chi exercise versus BINGO on fine motor functions in older adults," Medicine \& Science in Sports \& Exercise, vol. 51, no. Supplement, p. 313, 2019.

[19] H. W. Huang, N. Nicholson, and S. Thomas, "Impact of Tai Chi exercise on balance disorders: a systematic review," American Journal of Audiology, vol. 28, no. 45, pp. 1-14, 2019.

[20] V. Lee, C. H. Wu, Z. X. Lou et al., "Divergent and ultrahigh thermal conductivity in millimeter-long nanotubes," Physical Review Letters, vol. 118, no. 13, Article ID 135901, 2017.

[21] P. Y. Lee, T. G. Winfield, S. R. Harris et al., "Unloading knee brace is a cost-effective method to bridge and delay surgery in unicompartmental knee arthritis," BMJ Open Sport \& Exercise Medicine, vol. 2, no. 1, Article ID e000195, 2017.

[22] A. Fukumoto, K. Tajima, M. Hori, Y. Toda, S. Kaku, and H. Matsumoto, "Analgesic effect of S (+)-flurbiprofen plaster in a rat model of knee arthritis: analysis of gait and synovial fluid prostaglandin $\mathrm{E}_{2}$ levels," Journal of Pharmacy and Pharmacology, vol. 70, no. 7, pp. 929-936, 2018.

[23] P. Han, J. Peng, X. J. Ji et al., "The effect of arthroscopic synovectomy on refractory knee arthritis with popliteal cyst in 153 patients," Zhonghua Nei Ke Za Zhi Chinese Journal of Internal Medicine, vol. 58, no. 6, pp. 439-443, 2019.

[24] D. Y. Ponzio, U. A. M. Syed, K. Purcell et al., "Low prevalence of hip and knee arthritis in active marathon runners," The Journal of Bone and Joint Surgery, vol. 100, no. 2, p. 131, 2018.

[25] H. Zheng, B. Tulu, W. Choi, and P. Franklin, "Using mHealth app to support treatment decision-making for knee arthritis: patient perspective," eGEMs (Generating Evidence \& Methods to Improve Patient Outcomes), vol. 5, no. 2, p. 7, 2017.

[26] E. Rowbotham and A. Grainger, "Magnetic resonance imaging of arthritis of the knee," Seminars in Musculoskeletal Radiology, vol. 21, no. 2, pp. 113-121, 2017.

[27] M. D. Liu, X. K. Yang, F. Han et al., "Strategy for wound repair of skin and soft tissue defect and systematic rehabilitation treatment for functional reconstruction of patients with severe burn or trauma on knees," Chinese Journal of Burns, vol. 34, no. 5, pp. 266-270, 2018.

[28] J. H. V. Stan, M. P. Dijkers, J. Whyte et al., "The rehabilitation treatment specification system: implications for improvements in research design, reporting, replication and synthesis," Archives of Physical Medicine and Rehabilitation, vol. 100, no. 1, pp. 146-155, 2019.

[29] L. E. Simons, C. B. Sieberg, C. Conroy et al., "Children with chronic pain: response trajectories following intensive pain rehabilitation treatment," Journal of Pain Official Journal of the American Pain Society, vol. 19, no. 2, pp. 207-218, 2017. 